\title{
Radiofrequency catheter ablation of frequent premature ventricular contractions using ARRAY multi-electrode balloon catheter
}

\author{
Sergio Dubner, Claudio Hadid, Damian Azocar, Carlos Labadet, \\ Cecilia Valsecchi, Agustin Dominguez \\ Cardiology Department, Arrhythmias and Electrophysiology Service, \\ Clinica y Maternidad Suizo Argentina, Buenos Aires, Argentina
}

\begin{abstract}
Background: The noncontact mapping system facilitates the mapping of premature ventricular contractions (PVCs) and ventricular tachycardia (VT) using a 64-electrode expandable balloon catheter (ARRAY, St. Jude Medical). The aim of this study is to analyze the results and follow-up of the PVC ablation using this system.

Methods and results: Prospective and consecutive patients with frequent PVCs (6,000 or more) or monomorphic VT, suspected to be originated on the right ventricular outflow tract (RVOT), were included. The balloon catheter was positioned in the RVOT. Eighteen patients, 9 women, mean age 48 years (youngest/oldest 19-65) were included. Sixteen patients presented no structural heart disease. The origin of the arrhythmia was RVOT $(n=15)$, right ventricular inflow tract $(n=1)$, and left ventricular outflow tract $(n=2)$. Acute success was achieved in 15 patients; in 2 patients radiofrequency was not applied due to security reasons (origin site close to left coronary artery origin). The mean follow-up was 15 months (min. 4, max. 26); 13 patients presented abolition of the arrhythmia without drugs and 1 patient required antiarrhythmic drugs for arrhythmia control (previously ineffective). As an only complication, a femoral artery-venous fistula was observed.
\end{abstract}

Conclusions: The noncontact mapping system using a multielectrode balloon allows right ventricular arrhythmia treatment with a high rate of efficacy and safety. (Cardiol J 2016; 23, 1: $17-22)$

Key words: catheter ablation, frequent premature ventricular contractions, ARRAY balloon catheter, non-contact mapping, ventricular tachycardia

Editorial p. 1

\section{Introduction}

Premature ventricular contractions (PVCs) and ventricular tachycardia (VT) originating on the right ventricular outflow tract (RVOT) represent the most frequent ventricular arrhythmias in patients with normal hearts. VT from the RVOT's origin constitutes $80 \%$ of the idiopathic VT $[1,2]$.

Sustained, fast and repetitive VT can cause palpitations, syncope or other symptoms. Repetitive forms can lead to ventricular dysfunction [3].

Address for correspondence: Sergio J. Dubner, MD, FACC, Director Arrhythmias and Electrophysiology Service, Clinica y Maternidad Suizo Argentina and De Los Arcos Sanatorio, Arenales 2463, 3 A, 1124 Buenos Aires, Argentina, tel: (+5411) 4827 3654, e-mail: sdubner@cardiologyjournal.org

Received: 29.06.2015 Accepted: 01.09.2015 
PVCs may be asymptomatic or present as palpitations. Irrespective of symptoms, patients with very frequent PVCs ( $>10-15 \%$ of beats), can develop ventricular dilation with impaired systolic function, a form of non-ischemic dilated cardiomyopathy.

Drug therapy, mainly beta-blockers and calcium channel blockers, has limited effectiveness (25-50\%) in arrhythmia suppression. Other antiarrhythmic drugs, such as propafenone, flecainide and specially amiodarone, have better outcome but are associated with side effects. For this reason, radiofrequency ablation (RFA) is increasingly being used in these patients $[4,5]$ and has been suggested by some authors as first-line therapy due to its high success and low complication rate $[5,6]$.

Electroanatomic mapping systems are widely used to treat these arrhythmias. With this tool, an activation map is created by plotting local activation times using a color-coding scale. To do this, the chamber of interest (in this case right ventricle) must be explored moving the mapping catheter and collecting the local ventricular electrograms point by point once PVC occurs. Therefore, it requires the presence of frequent $\mathrm{PVCs}$ and/or prolonged episodes of VT to achieve an accurate mapping of the arrhythmia. Despite using this technology, ablation may not be successful in cases of infrequent PVCs, non-inducible or hemodynamically poorly tolerated arrhythmias in the laboratory. The non-contact mapping system (NCMS) is a self-expandable balloon catheter with 64 electrodes (Fig. 1), which allows simultaneous acquiring more than 3,000 virtual unipolar electrograms. Thus, mapping of arrhythmias with a single heartbeat can be performed $[7,8]$. There are limited data on outcomes in patients undergoing NCMS-guided RVOT ventricular arrhythmia ablation.

The aim of the study is to analyze the results and follow-up of radiofrequency catheter ablation in patients with PVCs and VT originating from RVOT with this technology.

\section{Methods}

Prospective and consecutive patients with frequent PVCs or monomorphic VT evaluated in our institution were included. Inclusion criteria were monomorphic PVCs $>6,000 / 24$ h or monomorphic VT, with left bundle branch block morphology on 12-lead electrocardiogram (ECG) or 12-lead Holter recording and suspected origin at RVOT, scheduled for catheter ablation due to frequent symptoms, refractoriness or intolerance to antiarrhythmic drugs, or patient preference.

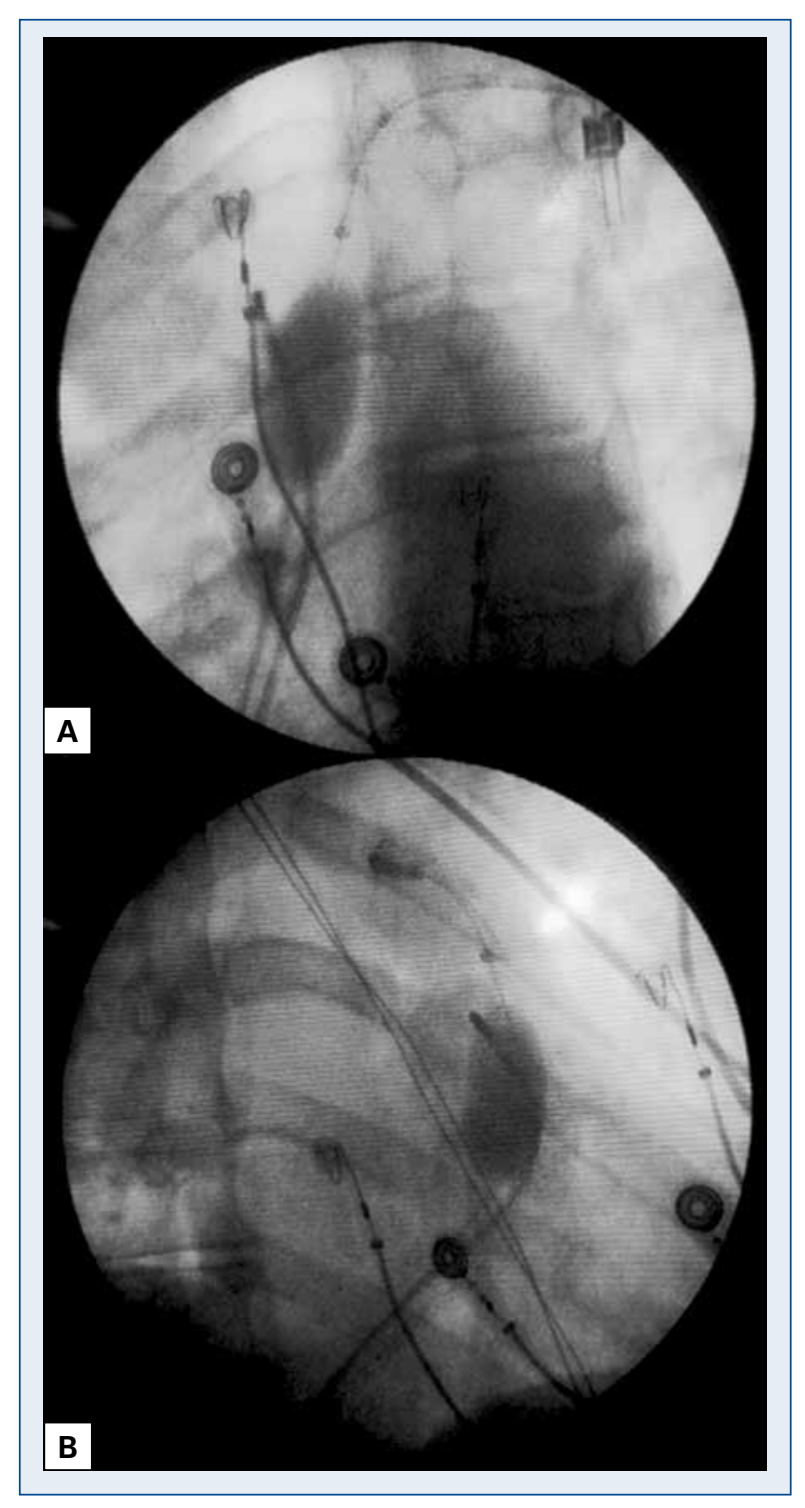

Figure 1. Balloon catheter in the right ventricular outflow tract, high profile filled with iodide contrast to allow X-ray view; A. Left anterior oblique view; B. Right anterior oblique view.

The balloon catheter (EnSite Array, St. Jude Medical, St. Paul, Minnesota, USA) was introduced via the right femoral vein through a $10 \mathrm{Fr}$ introducer and under fluoroscopic view over a 0.35 " wire that had been placed in the pulmonary artery. The deflated balloon (low profile) was advanced "over-the-wire" to the RVOT. Patients were heparinized in order to achieve an activated clotting time target between $300 \mathrm{~s}$ and $400 \mathrm{~s}$. Once positioned in the RVOT, the balloon was inflated (high profile) and filled with iodinated contrast for radiological visualization (Fig. 1). Subsequently, the 3-dimensional geometry of the right ventricle 


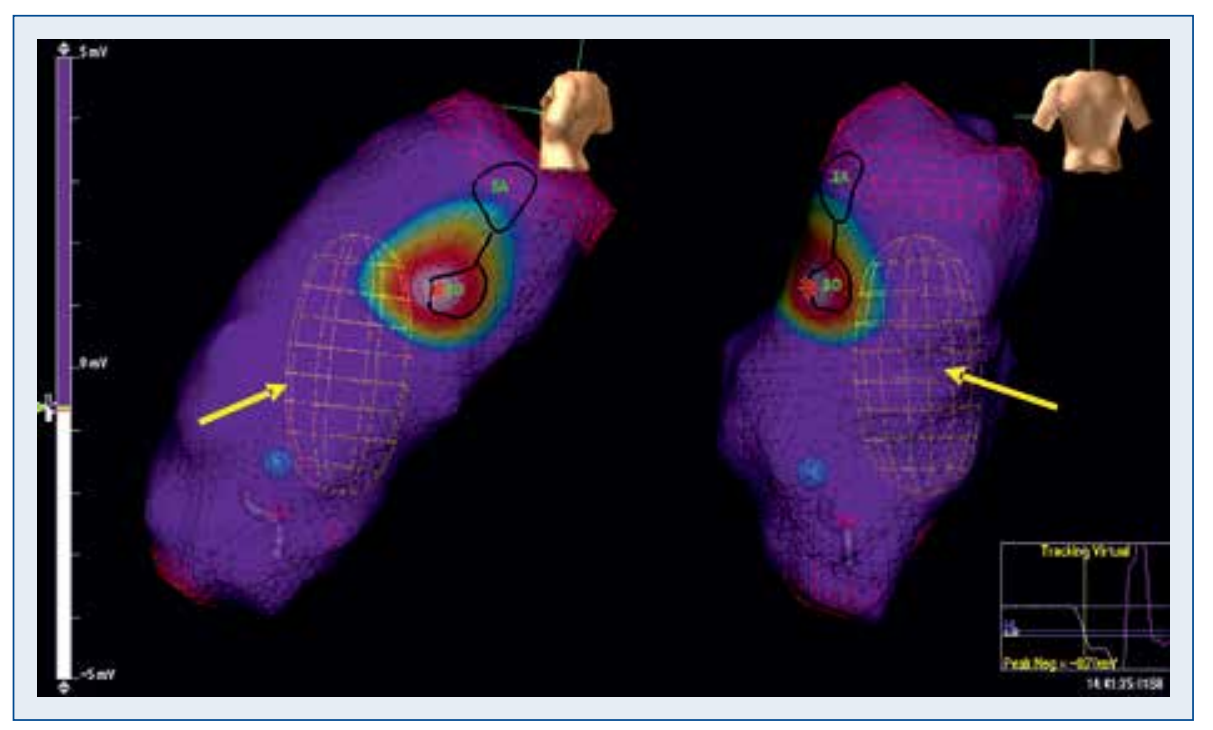

Figure 2. Right ventricular outflow tract's activation map where earliest activation (EA) is observed following myocardial activation (BO, break out). Yellow arrows show the balloon catheter.

was acquired using the ablation catheter. Important anatomic structures, such as the His bundle and pulmonary valve were identified and labeled. An activation map was created based on recorded balloon catheter unipolar electrograms from spontaneous PVCs that matched the clinical arrhythmia. Identification of the site of earliest activation (EA) as well as the output of the activation front to the rest of myocardium (break out [BO]) was obtained with one single beat. This process was repeated with another 3 PVCs to confirm the reproducibility of the map (Fig. 2).

General anesthesia was used in some cases. Due to its intrinsic effect to reduce or even abolish ectopic activity, in some cases we used mild sedation or local anesthesia only if the patient consented. Isoproterenol or programmed ventricular stimulation was used in patients without spontaneous arrhythmia during the procedure. To avoid arrhythmia induction caused by contact with the endocardium, all other catheters were withdrawn to the right atrium at the time of arrhythmia mapping. 12-lead concordance between the PVCs/VT recorded in the laboratory and those presented in the daily clinic was carefully assessed.

Then, unipolar electrograms from the multielectrode balloon catheter were used to identify the site of origin of the arrhythmia and visualize the spread of the electrical impulse from the EA to $\mathrm{BO}$ and then to the rest of the myocardium. The focus origin was determined by the QS pattern in the unipolar electrograms obtained with the balloon catheter. A QS pattern on a unipolar electrogram identifies the start depolarization site, from which the activation front centrifugally spreads away.

Radiofrequency application was addressed to both the EA and the BO points using $4 \mathrm{~mm}$ nonirrigated tip catheter $\left(50 \mathrm{~W}, 60^{\circ} \mathrm{C}\right)$ with applications of $60 \mathrm{~s}$ until abolition of the arrhythmia. If a second focus of arrhythmia was observed after the first focus was targeted, a similar identification process and ablation was performed.

Acute success was defined as the sustained disappearance (total abolition) for at least $30 \mathrm{~min}$ of the spontaneous PVCs/VT or the reduction greater than $99 \%$. The occurrence of the arrhythmia after this period was considered recurrence. Failure in abolishing the arrhythmia was considered as acute failure of the procedure.

Patients remained hospitalized until the next morning in coronary care unit with continuous ECG recording and a transthoracic echocardiography was performed before and after the intervention.

During the follow-up all patients were evaluated every 15/30 days (first 2 months) and every 4 months later, with routinely clinical controls, ECG and 24-h Holter recording during the first year after the procedure.

The study was approved by the local bioethical committee and all patients signed an informed consent.

\section{Results}

Between February 2012 and December 2014, 18 patients ( 9 women) with a mean age of 48 years 
(min. 19, max. 65) were included. Only 2 patients had structural heart disease (nonischemic dilated cardiomyopathy and coronary artery disease). Most patients had frequent PVCs $(\geq 6,000 / 24 \mathrm{~h})$, $4(25 \%)$ patients presented non sustained VT and 3 patients presented sustained monomorphic VT. The average arrhythmic density before the procedure was $12,350 \mathrm{PVCs} / 24 \mathrm{~h}$ (min. 6,366, max. 25,671). The mean number of unsuccessful antiarrhythmic drugs used per patient was 1.4 (min. 0, max. 5). In 4 patients, the RFA was the first-line treatment due to patients' choice. The arrhythmia focus was identified in the RVOT in $15(83 \%)$ patients and right ventricular inflow tract in 1 patient. In the remaining 2 patients, the origin was identified in the left ventricular outflow tract near the origin of the left coronary artery; in both of them radiofrequency was not applied for safety reasons. Acute success, measured with abolition of the arrhythmia and the absence of symptoms, was achieved in 15/16 (93.7\%) patients in whom radiofrequency was delivered (1 failure) and in all but 3 of the total number of patients $(15 / 18,83 \%)$. In 5 patients, 2 or more ventricular ectopic foci were found and ablated in the RVOT. In the patient with failed RFA, the focus was identified at the interventricular septum and radiofrequency was delivered from both sides (right and left) with no change in arrhythmia burden.

The average procedure time was $186 \mathrm{~min}$ (min. 120, max. 240) and mean fluoroscopy time was $37 \mathrm{~min}$ ( $\min .21$, max. 59). There were no acute hemodynamic procedure complications and all patients were discharged within $24 \mathrm{~h}$. Two patients presented incomplete right bundle branch block that persisted during the follow-up. One patient developed a femoral artero-venous fistula, with surgical resolution.

During the follow-up (average 15 months, min. 4, max. 26) 13 of 16 patients in whom radiofrequency was delivered $(81 \%)$ were free from both arrhythmia and symptoms in a drug-free state. Two patients with acute RFA success had arrhythmia recurrence that was controlled with sotalol (previously ineffective) in one patient and with a second ablation procedure in the other patient. The patient with failed RFA was kept on antiarrhythmic drugs during the follow up, with poor response.

\section{Discussion}

The main result of this series is the high success rate and safety of NCMS in the patient population with outflow tract ventricular arrhythmia. Acute success was achieved in $93.7 \%$ of pa- tients in whom radiofrequency was delivered with 1 complication (femoral artero-venous fistula). During the follow-up (average 15 months) 13 patients were free from arrhythmia without antiarrhythmic drug therapy and only 2 patients with acute success had recurrence. In 1 patient, the ablation procedure appears to have modified the arrhythmia substrate since PVC burden could be controlled by previously ineffective antiarrhythmic drugs. This may be interpreted as partially successful ablation.

The mapping of the arrhythmia focus with a single heartbeat deserves a special comment. Activation maps from 3 additional PVCs were performed to confirm the focus location. This means that with less than 5 PVCs the target for ablation can be successfully identified, without need for a point-by-point mapping of the RVOT. Together with the high success rate of the RFA, this finding suggests the high accuracy of this technique for identification of the target site for ablation.

These results highlight the importance of patient selection for the use of this system. The best candidates are those with suspected origin in the RVOT (left bundle branch block morphology, inferior frontal axis, R/S transition > V3) $[9,10]$. This system has also been used in other right ventricle sites (right ventricular inflow tract in case number 6 of our series), as well as in right atrium [11], left atrium [10] and even in the left ventricle [12]. The high effectiveness of this system in our series is in concordance with other published studies [12-15]. Tsuchiya [12] have reported the use of the NCMS in 55 patients with ventricular arrhythmia. The RVOT was the most frequent location of origin and successful ablation achieved in all but 3 patients with epicardial origin. Friedman et al. [14] have reported the success of ablation in 9 of 10 patients with non-inducible VT originating from the RVOT. Ling et al. [16] reported superiority of RFA over antiarrhythmic medication in arrhythmia suppression in patients with RVOT PVCs, in a prospective randomized study. Zhang et al. [17] found NCMS-guided RVOT catheter ablation highly effective, and clinical success was best achieved by ablating the EA site, rather than $\mathrm{BO}$ site.

Improvement of ejection fraction is another benefit after ventricular arrhythmias disappear. In the meta-analysis performed by Lamba et al. [18], left ventricular ejection fraction (LVEF) was reported in 5 of the 14 studies evaluated, which included 108 patients. Radiofrequency catheter ablation significantly improved LVEF by a mean of 10.36 (confidence interval 8.75-11.97, $\mathrm{p}<0.00001$ ) in patients with frequent PVCs from the RVOT. 
Despite the efficacy of this system, there are some limitations to this technique. The progression of the guidewire and the deflated balloon to the pulmonary artery is not a simple task and may require a significant fluoroscopy time. In our case it was possible to successfully carry out such maneuver in every patient, but in some cases it took up to $30 \mathrm{~min}$. We observed a reduction of total and fluoroscopy times when comparing recent cases with the initial ones (learning curve) [19]. Another limitation of the method is related to the distance between the central axis of the balloon catheter and the focus of the arrhythmia. When the distance between them is greater than $4 \mathrm{~cm}\left(1^{37} / 64\right.$ inches $)$, the confidence of the activation map is impaired, and chances of applying radiofrequency in a wrong site are higher, and hence, efficiency is reduced. This is particularly important in arrhythmias originating in sites other than the RVOT (left ventricle, other areas of the right ventricle). To avoid this problem it is essential a deepest evaluation of the 12-lead ECG while planning the procedure. In one of our cases it was noted that the focus was located in the region of the tricuspid valve, so we pulled-down the balloon catheter closer to the arrhythmia focus, to ablate the basal region of the right ventricle. Another limitation of the method is the contact of the catheter balloon with the endocardium in certain circumstances, especially when using isoproterenol. It may generate an ectopic activity different from the clinical one. Despite the considerable size of the inflated balloon catheter, we have not observed any hemodynamic side effect. Schneider et al. [20] found similar results in 20 consecutive pediatric patients with idiopathic VT. The region of origin was identified in all of them, and the site of earliest activation with a QS pattern of the unipolar electrograms was guided for ablation. The authors concluded that noncontact mapping can safely and effectively be used to map and guide catheter ablation of the tachycardia substrate of idiopathic VT in pediatric patients. Finally, the higher cost than other mapping systems is an important issue to be taken into account.

\section{Limitations of the study}

The small sample size and the rigorous patient selection may not be representative of rare forms of these arrhythmias (i.e. epicardial origin) or the occurrence of rare complications [19]. Since it is a single-center study, we cannot compare this result with different groups.
In 5 patients, we identified more than one focus of origin of the PVCs. However, radiofrequency applications can generate small shifts in arrhythmia exit sites and/or spread of electrical activation.

\section{Conclusions}

Noncontact mapping system with multielectrode balloon catheter allows an excellent and precise identification of the site of origin of the right ventricular arrhythmia. This mapping method allows obtaining a high rate of effectiveness of the ablation procedure, with high safety. Further studies with more patients and the comparison with antiarrhythmic drugs and other forms of catheter mapping can confirm these findings.

\section{Conflict of interest: None declared}

\section{References}

1. Garcia Civera R, Ruiz Granell R, Morell Cabedo S, Sanjuan Máñez R, Martinez León J, Botella Solana S, López Merino V. Electrofisiología cardiaca clínica y ablación. McGraw-Hill Interamericana, 1999: 327-343.

2. Pytkowski M, Maciąg A, Sterliński M et al. Novel algorithm for arrhythmogenic focus localization in patients with right ventricular outflow tract arrhythmias. Cardiol J, 2014; 21: 284-292.

3. Yarlagadda RK, Iwai S, Stein KM et al. Reversal of cardiomyopathy in patients with repetitive monomorphic ventricular ectopy originating from the right ventricular outflow tract. Circulation, 2005; 112: 1092-1097.

4. Aliot EM, Stevenson WG, Almendral-Garrote JM et al. EHRA/ /HRS Expert Consensus on catheter ablation of ventricular arrhythmias. Heart Rhythm,2009; 6: 886-933.

5. Gant López J, Labadet C, González JL et al. Primer Registro Argentino de Ablación con Catéter. Rev Argent Cardiol, 2011; 79: 117-124.

6. Ferrero de Loma-Osorio A, Gil-Ortega I, Pedrote-Martínez A. Spanish Catheter Ablation Registry. 13th Report of the Spanish Society of Cardiology Working Group on Electrophysiology and Arrhythmias (2013). Rev Esp Cardiol, 2014; 67: 925-935.

7. Ribbing M, Wasmer K, Mönnig G et al. Endocardial mapping of right ventricular outflow tract tachycardia using noncontactactivation mapping. J Cardiovasc Electrophysiol, 2003; 14: 602-608.

8. Schilling RJ, Peters NS, Davies DW. Feasibility of a noncontact catheter for endocardial mapping of human ventricular tachycardia. Circulation, 1999; 99: 2543-2552.

9. Miles WM. Idiopathic ventricular outflow tract tachycardia: Where does it originate? J Cardiovasc Electrophysiol, 2001; 12: 536-537.

10. Cohen TJ, Juang G, Daggubati R. Utility of non-contact threedimensional mapping of the left atrium for ablation of left atrial tachycardia. J Invasive Cardiol, 2004; 16: 100-101.

11. Wieczorek M, Salili AR, Kaubisch S, Hoeltgen R. Catheter ablation of non-sustained focal right atrial tachycardia guided by virtual non-contact electrograms. Europace, 2011; 13: 876-882. 
12. Tsuchiya T. Three-dimensional mapping of cardiac arrhythmias — string of pearls. Circulation, 2012; 76: 572-581.

13. Díaz Infante E, Cózar León R, Borrego I, Maldonado J, Nieto P, Cruz Fernández JM. Ablation of premature ventricular contractions originating in the right ventricular outflow tract using noncontact mapping. Rev Esp Cardiol, 2011; 64: 1198-1201.

14. Friedman PA, Asirvatham SJ, Grice $\mathrm{S}$ et al. Noncontact mapping to guide ablation of right ventricular outflow tract tachycardia. J Am Coll Cardiol, 2002; 39: 1808-1812.

15. Villacastin J, Perez CN, Moreno J, Alvarez L, Moreno M, Quintana J. Percutaneous epicardial radiofrequency ablation of idiopathic ventricular tachycardia. Rev Esp Cardiol, 2005; 58: 100-104.

16. Ling $Z$, Liu $Z$, Su L et al. Radiofrequency ablation versus antiarrhythmic medication for treatment of ventricular premature beats from the right ventricular outflow tract: Prospective randomized study. Circ Arrhythm Electrophysiol, 2014; 7: 237-243.
17. Zhang $\mathrm{F}$, Yang $\mathrm{B}$, Chen $\mathrm{H}$ et al. Noncontact mapping to guide ablation of right ventricular outflow tract arrhythmias. Heart Rhythm, 2013; 4: 1895-1902.

18. Lamba J, Redfearn DP, Michael KA, Simpson CS, Abdollah H, Baranchuk A. Radiofrequency catheter ablation for the treatment of idiopathic premature ventricular contractions originating from the right ventricular outflow tract: A systematic review and meta-analysis. Pacing Clin Electrophysiol, 2014; 37: 73-78.

19. Martirosyan M, Kiss A, Nagy-Baló et al. Learning curve in circular multipolar phased radiofrequency ablation of atrial fibrillation. Cardiol J, 2015; 22: 260-265.

20. Schneider HE, Kriebel T, Jung K, Gravenhorst VD, Paul T. Catheter ablation of idiopathic left and right ventricular tachycardias in the pediatric population using noncontact mapping. Heart Rhythm, 2010; 7: 731-739. 